\title{
PREDICTORES GLOBALES DE LA PERFORMANCE DE ESTUDIANTES MILITARES
}

\section{GLOBAL PREDICTORS OF THE MILITARY STUDENTS' PERFORMANCE}

\author{
Pablo Domingo Depaula* \\ Secretaría de Investigación del Colegio Militar de la Nación, Argentina
}

\begin{abstract}
Resumen: El estudio aborda transversalmente una muestra de 400 estudiantes militares, considerando la evaluación de su performance resultante de la formación e instrucción recibida. Efectuándose un análisis de regresión lineal, se indagó ad hoc el nivel predictivo de determinadas variables sobre el rendimiento alcanzado. Se concluye que las personalidades responsables, con fuerte voluntad e inteligencia cultural reflexiva-motivacional constituyen predictores actuales del óptimo rendimiento; asimismo, durante las prácticas iniciales de conducción, un estilo de liderazgo laissez faire predice negativamente la performance, mientras que la inteligencia cultural cognitiva resulta un genuino predictor prospectivo operando en etapas de conducción de grupo y subinstrucción supervisada.
\end{abstract}

Palabras clave: estilos de personalidad; rendimiento; inteligencia cultural; competencias

\begin{abstract}
The aim of this research is to evaluate the performance resulting from the training of a sample composed by 400 military students. A cross-sectional study and a linear regression analysis, aimed at determining if certain variables could predict the students' performance, were performed. It can be concluded that responsibility, strong will, and a reflexive-motivational cultural intelligence can predict optimal performance. On the other hand, a laissez-faire leadership style indicates a negative performance while the cognitive cultural intelligence is a prospective predictor in group leadership and in supervised sub-training.
\end{abstract}

Keywords: personality styles; performance; cultural intelligence; skills

\begin{abstract}
* La investigación en que se sustenta este artículo recibió el tercer premio a jóvenes investigadores "Dr. Jorge Galeano Muñoz" en el marco de las XX Jornadas Uruguayas de Psicología, "Aportes de la Psicología a la Educación y a la Convivencia", organizadas por la Sociedad de Psicología del Uruguay, 2011. El texto que sigue corresponde a una "versión completa" del trabajo presentado en el evento científico mencionado.
\end{abstract}

\section{INTRODUCCIÓN}

Gran parte de las academias militares actuales consideran significativas ciertas características individuales involucradas en la formación y entrenamiento de sus estudiantes, las que exceden, por ejemplo, el área relativa a los rasgos de la personalidad. Tales academias europeas y americanas sobre todo, han implementado rigurosos procesos de selección reclutando estudiantes, formándolos especializadamente y entrenándolos intensamente para operar en escenarios internacionales; al tiempo que desarrollan programas específicos de incorporación internacional de alumnos europeos, con el objetivo de promover la interacción entre cadetes locales y extranjeros, en virtud de facilitar el desarrollo de competencias militares que permitan el desempeño eficaz en diversas regiones del mundo.
Bajo este panorama, la relevancia de la educación cultural de los cuadros militares ha sido enfatizada por diversos autores (McFarland, 2005; Rubinstein, 2003; Schwerzel, 2005; Skelton \& Cooper, 2005). Así, resulta necesario examinar críticamente los programas de formación y desarrollo del liderazgo militar y los predictores del rendimiento de estudiantes militares.

Sobre la base de estos modelos de formación transcultural, gran cantidad de líderes militares y soldados despliegan operaciones de apoyo o mantenimiento de la paz en representación de Naciones Unidas (NN.UU.), cuyo accionar difiere a las guerras tradicionales, implicando un mayor requerimiento de trabajo con otras culturas nacionales y organizaciones no militares, motivos por los que se justifica la transformación y reorganización de las fuerzas militares modernas hacia la adopción de nuevos roles en un vasto 
espectro de operaciones ( $\mathrm{Ng}$, Ramaya, Teo \& Wong, 2005). En suma, el ejercicio del liderazgo militar implica la identificación de una conciencia cultural o competencia cultural, necesaria para el óptimo despliegue de operaciones militares en contextos culturales diversos (Graen \& Hui, 1999; Gurstein, 1999; Shamir \& Ben-Ari, 1999; Yukl, 1999 citados por $\mathrm{Ng}$ et al., 2005).

El abordaje de los factores que predicen el rendimiento en instituciones militares configura una temática de gran relevancia, justificando la evaluación psicológica de los estudiantes a fin de analizar y reelaborar los programas educativos, identificar jóvenes en riesgo de fracaso a causa de factores psicopatológicos, predecir el rendimiento exitoso (ya sea, en la instrucción militar como en la formación académica), evaluar el costo económico y el impacto social de la carrera y seleccionar adecuadamente los recursos humanos (Castro Solano, 2005).

Conforme a investigaciones precedentes realizadas por Castro Solano y Casullo (2001), en general aquellos estudiantes que habían manifestado problemas durante su entrenamiento, pero que lograron afrontarlos, se diferenciaban del resto en su estilo de personalidad. Estos individuos, que registraban un menor rendimiento a lo largo de su formación, eran extrovertidos y buscaban frecuentemente excitación, estimulación y atención del entorno; dichas características de personalidad, resultan poco adaptativas para el entorno militar. Por otro lado, otro estudio desarrollado por los mismos autores determinó que, en lo que respecta a la variable motivación para el aprendizaje, aquellos estudiantes con menor interés en el contenido académico estaban orientados por una motivación más extrínseca y, por lo tanto, obtenían bajos rendimientos (Castro Solano \& Casullo, 2002).

Bartone (1999), miembro de la academia militar West Point Army, en la pasada década argumentaba que "futuros oficiales militares deben ser altamente resilientes, ingeniosos, y rápidos para ajustarse a situaciones que cambian rápidamente" (p. 1), reconsiderando entonces el rol de los rasgos de la personalidad normal que podrían influir sobre el rendimiento de los futuros líderes militares, puesto que hasta el momento, las investigaciones se habían centrado en la consideración de los rasgos patológicos de la personalidad. En función de dicho objetivo, los resultados de un estudio realizado por Bartone (1999) han sugerido que variables cognitivas, emocionales y de la personalidad constituyen un modelo integrado que opera influyendo sobre el desarrollo y la performance del líder.

En suma, factores como la capacidad adaptativa del sujeto, la motivación o los estilos de personalidad, adquieren preponderancia en la explicación del éxito académico. Asimismo, las calificaciones de los pares o superiores, sistema bastante utilizado en las Fuerzas Armadas, permiten predecir de manera más acabada el desempeño en el puesto de trabajo futuro (Braun, Wiegand \& Aschenbrenner, 1991; Castro Solano, 2005).

En general, Castro Solano (2005) establece que existen diversos predictores del rendimiento académico en ámbitos militares, tales como los relacionados con: las competencias intelectuales, la motivación, los intereses, los rasgos de personalidad y las variables del contexto.

En relación a las variables del contexto anteriormente mencionadas, existirían otros factores de importancia para la formación de los estudiantes, que podrían predecir el rendimiento general y que emergerían del atravezamiento del fenómeno de la globalización sobre la profesión del militar contemporáneo; por ejemplo, la inteligencia cultural, constructo que originalmente aludía a ciertas capacidades personales para adaptarse de manera efectiva a nuevos contextos culturales (Earley \& Ang, 2003). Su importancia está vinculada al potencial desenvolvimiento del militar en ámbitos de alta incertidumbre y/o conflicto.

La definición conceptual más contundente acerca de la inteligencia cultural es aportada por Thomas et al. (2008) quienes postulan que dicho constructo constituye un sistema de interacción de conocimientos y habilidades conectadas mediante "metacognición cultural" (p. 127), permitiéndole a las personas adaptarse, seleccionar y configurar los aspectos culturales de su ambiente. Este factor constituye el centro de gravedad hacia el logro de la adaptación cultural, representando una función de enlace entre conocimientos y habilidades culturales. 


\section{OBJETIVOS}

- Analizar jerárquicamente en qué grado la inteligencia cultural, la personalidad y la motivación para el aprendizaje, resultan predictores del rendimiento general de los estudiantes militares.

- Analizar los niveles de jerarquía en que la inteligencia cultural, los estilos de liderazgo y los valores humanos en el trabajo, predicen el rendimiento general de los estudiantes militares.

\section{MATERIAL Y MÉTODOS}

\subsection{Tipo de estudio}

Se diseñó una investigación correlacional delimitando las siguientes variables principales: inteligencia cultural, personalidad, motivación para el aprendizaje, estilos de liderazgo y valores humanos que presentaban los estudiantes militares.

\subsection{Participantes}

La muestra del estudio estuvo integrada por 400 cadetes aspirantes a oficiales del Ejército Argentino, que durante los meses de agosto y septiembre del año $2008^{1}$ cursaban una Licenciatura en una institución de formación militar. Se seleccionó una muestra no probabilística por cuotas según el año en curso, considerándose 100 cadetes de cada uno de los cuatro años de la carrera. El $94,3 \%(n=377)$ de los cadetes eran de sexo masculino y el $5,8 \%(n=23)$ de sexo femenino. La edad promedio era de 21,71 años $(S D=2,37$ años).

Por otro lado, el $98,2 \%$ de los estudiantes ( $n=393$ ) eran nacidos en Argentina, en las siguientes regiones geográficas: un $45,3 \%$ de los cadetes $(n=181)$ eran nacidos en la región denominada Pampa o Llanura Pampeana (la que incluye la capital del país, entre otras provincias del interior), $14,5 \%(n=58)$ en el Noroeste Argentino, $14,3 \%(n=57)$ en la Mesopotamia, $8,5 \%(n=34)$ en las Sierras Pampeanas, 7,8 ( $n$ $=31)$ en la región de Cuyo, 6,3\% $(n=25)$ eran oriundos de la Patagonia y $1,8 \%(n=7)$ de la

1. El tiempo transcurrido desde la realización de la investigación hasta su publicación se explica porque el presente estudio corresponde a algunos de los objetivos específicos abordados en la Tesis de Maestría en Psicología Organizacional realizada por Depaula (2010), la cual debía contener información inédita. región del Chaco. El resto de los cadetes (1,8\%; $n=7$ ) habían nacido en el exterior del país. La totalidad de los estudiantes $(N=400)$ residían en Buenos Aires al momento de la recolección de datos, dado el régimen de internado semanal que requiere la cursada académica e instructiva en este instituto de formación militar, localizado al oeste del conurbano bonaerense.

Con respecto al arma o especialidad de la que formaban parte los estudiantes (por elección voluntaria o por orden de mérito -cuando existe una gran cantidad de cadetes que eligen determinadas armas o especialidades-) el $25 \%$ ( $n=100)$ pertenecía al Cuerpo Comando, es decir aquellos cursantes del primer año de la carrera que aún no han elegido arma o especialidad, dado que tal determinación se lleva a cabo una vez que alcanzan el segundo año. El $17,5 \%(n=70)$ de los cadetes pertenecía al arma de Infantería, el $14 \%(n=56)$ a Caballería, el $12,5 \%(n=50)$ integraba la especialidad de Ingenieros, el 11,8\% $(n=47)$ pertenecía al arma de Artillería, el 9,5\% $(n=38)$ integraba la especialidad de Comunicaciones, el $6 \%$ $(n=24)$ a la de Intendencia y el 3,8\% $(n=15)$ a la de Arsenales.

\subsection{Instrumentos}

Encuesta Sociodemográfica (Depaula, 2010): Corresponde a un instrumento diseñado ad hoc para el presente estudio, cuyo propósito era indagar datos demográficos de los participantes tales como el sexo, la edad, el lugar de nacimiento, el año de cursada de una Licenciatura en una institución de formación militar, y por último el arma o la especialidad que integraban los cadetes en el instituto.

Cultural Intelligence Scale (CQS) (Ang et al., 2007): La CQS corresponde a un cuestionario que consta de 20 ítems, que evalúan las cuatro dimensiones que componen la inteligencia cultural (Earley \& Ang, 2003), a saber, la dimensión metacognitiva, que refleja los procesos mentales que los individuos utilizan para adquirir y comprender el conocimiento cultural, incluyendo el control de conocimientos y pensamientos relativos a la cultura, e integra capacidades que incluyen la planificación, monitoreo y revisión de modelos mentales relativos a normas culturales pertenecientes a países o grupos de personas; la dimensión cognitiva, 
que evalúa el conocimiento de normas, prácticas y convenciones adquiridas en diferentes culturas, producto de la educación y las expe riencias personales, incluyendo conocimientos relativos a los sistemas económicos, legales y sociales de diversas culturas y subculturas, y conocimientos acerca del marco de valores culturales que distingue a cada cultura en particular; la dimensión motivacional, que refleja la capacidad de dirigir la atención y la energía hacia el aprendizaje y funcionamiento en situaciones caracterizadas por diferencias culturales, operando como un agente de control de afectos, cogniciones y conductas que facilitan el logro de objetivos. Por último, la dimensión conductual, que permite evaluar la capacidad para exhibir apropiadamente acciones verbales y no-verbales, interactuando con personas de diferentes culturas, a través de valores culturales propios de contextos específicos e incluyendo un vasto repertorio de conductas. Los reactivos se responden mediante una escala de tipo Likert con 7 opciones de respuesta, pero a fin de reducir los tiempos de administración, en este estudio, el formato contempló 5 opciones cuyo escalamiento presentaba las categorías entre Muy en desacuerdo/En parte en desacuerdo/Ni de acuerdo ni en desacuerdo/En parte de acuerdo/ Muy de acuerdo. El cuestionario fue diseñado por sus autores para ser auto-administrado en forma individual o colectiva.

Por otro lado, en lo que respecta a los criterios de validez y confiabilidad de la prueba, (Depaula, 2010), traduciendo al idioma castellano la versión del instrumento propuesta por Ang et al. (2007), ha realizado nuevos análisis factoriales exploratorios de componentes principales con rotación Promax, obteniendo una solución factorial definitiva que consta de tres factores que explican el $50,936 \%$ de la varianza (Test de Esfericidad de Bartlett $=2284,104 ; p<0,01$ ) (Índice Kaiser Meyer Olkin $=0,842$ ). Este proceso fue llevado a cabo de forma iterativa, eliminándose tres de los ítems provenientes de la escala original; dado que inicialmente se presentaban niveles de fiabilidad significativamente bajos en uno de los factores (integrado por dos ítems originales) resultantes de la estructura factorial preliminar ( $\alpha$ de Cronbach $=0,2773$ ), al tiempo que en la estructura factorial definitiva se eliminó un ítem que presentaba cargas factoriales similares en más de un factor.
Tales resultados, han mostrado que la última versión del instrumento presenta propiedades psicométricas aceptables. En efecto, ha arrojado niveles meritorios de fiabilidad ( $\alpha$ de Cronbach $=0,8149$ ). De un posterior análisis de la fiabilidad realizado a través del modelo de dos mitades (split), también han resultado niveles aceptables de fiabilidad (valor $\alpha$ para la parte $1=0,7771$; valor a para la parte $2=0,7111$ ).

En virtud de los análisis estadísticos mencionados, la escala utilizada en el presente estudio se halla integrada por las siguientes dimensiones: inteligencia cultural reflexivamotivacional ( $\alpha$ de Cronbach $=0,7728$ ); inteligencia cultural cognitiva ( $\alpha$ de Cronbach $=0,7898)$; inteligencia cultural conductuallingüística ( $\alpha$ de Cronbach $=0,7935$ ).

Big Five Inventory (BFI) (Castro Solano \& Casullo, 2001; John, 1990): Este cuestionario corresponde a una operacionalización representativa del modelo teórico big five, que evalúa los cinco factores de la personalidad a saber: extraversión, responsabilidad, neuroticismo, agradabilidad y apertura a la experiencia. Se trata de un instrumento auto-administrable (en forma individual o colectiva) dispuesto de 44 ítems a valorarse a través de una escala con formato Likert de 5 opciones de respuesta, cuyo escalamiento presenta las categorías: Muy en desacuerdo/En parte en desacuerdo/Ni de acuerdo ni en desacuerdo/En parte de acuerdo/ Muy de acuerdo.

En cuanto a las propiedades psicométricas de este instrumento, Castro Solano y Casullo (2001) validaron el BFI en Argentina, para la valoración de la personalidad en población adolescente, desarrollando un estudio del que participaron sujetos civiles y cadetes militares (ingresantes a la carrera respectiva o transitando los últimos niveles de formación). Primeramente, los autores verificaron la homogeneidad de cada una de las escalas del BFI realizando un análisis de la fiabilidad a través del cálculo del coeficiente alfa de Cronbach, el cual arrojó los siguientes resultados: 'Población cadetes argentinos' $(n=360)$, extraversión: 0,71; agradabilidad: 0,55 ; responsabilidad: 0,69; neuroticismo: 0,73 ; apertura a la experiencia: 0,69 . 'Población civiles argentinos' ( $n=276)$, extraversión: 0,68; agradabilidad: 0,66 ; responsabilidad: 0,70 ; neuroticismo: 0,74; apertura a la experiencia: 0,77. 
Por otro lado, un análisis factorial de los ítems a través del método Varimax permitió obtener cinco factores que explicaban el $45 \%$ de la varianza de las puntuaciones, verificando que los ítems del BFI, tanto en población militar como civil, se agrupan según las cinco grandes dimensiones del enfoque big five (Castro Solano, 2005).

Escala de Metas de Aprendizaje, Población Militar (EMA-M) (Castro Solano \& Casullo, 2002): Consiste en una operacionalización del modelo de motivación para el aprendizaje postulado por Alonso Tapia (1992, 1997 citado por Castro Solano, 2005), el cual considera que las metas del aprendizaje pueden ser internas y/o externas. En la misma línea, los aportes de Bandura (1999) vinculados a la auto-eficacia y la motivación académica, resultan centrales para la evaluación y el análisis de esta variable.

Este instrumento puede ser utilizado únicamente en población de estudiantes militares, ya que las frases reflejan el contenido relacionado con la educación académica aplicada en tal ámbito. El cuestionario fue diseñado para ser autoadministrado, pudiendo administrarse en forma individual o colectiva; los reactivos se responden mediante una escala con formato Likert con 5 opciones de respuesta, cuyo escalamiento presenta las categorías Muy en desacuerdo/En parte en desacuerdo/Ni de acuerdo ni en desacuerdo/En parte de acuerdo/Muy de acuerdo.

Las dimensiones evaluadas a través de la prueba se definen conceptualmente de la siguiente forma: metas en relación con el aprendizaje y el dominio de los conocimientos, es decir que se aprende por el deseo de aprender en sí mismo, porque tiene un valor en sí mismo, por satisfacción personal o por el deseo de dominar algún aspecto del conocimiento sin buscar obtener algo a cambio; metas aversivas externas, significando que las consecuencias producto del estudio son las cuestiones importantes a considerar y no el aprendizaje en sí mismo, manifestándose aversión o repulsión por el objeto de conocimiento; y por último, metas de lucimiento, las que indican que se aprende para ser aceptado, para quedar bien con los demás, ya sea con profesores o instructores militares, es decir ser valorado por sus superiores, por ejemplo.

Los autores del instrumento llevaron a cabo un análisis factorial cuya solución explicaba el
$60 \%$ de la varianza de las puntuaciones. La estructura factorial obtenida, presentaba un primer factor correspondiente a metas en relación con el aprendizaje y el dominio de los conocimientos, un segundo factor relacionado con las metas aversivas externas, y un tercer factor vinculado a las metas de lucimiento; acto seguido, verificaron la estructura factorial obtenida a través de un análisis factorial confirmatorio, encontrando, en población militar más que en civil, un mejor ajuste de la estructura factorial al modelo teórico elegido. Por último, analizaron la fiabilidad del instrumento calculando el coeficiente alfa de Cronbach, obteniendo fiabilidades satisfactorias para cada uno de los tres factores, a saber: factor 1, 'metas en relación con el aprendizaje y dominio' (alfa $=0,70$ ); factor 2 , 'metas aversivas externas' (alfa $=0,73$ ); factor 3 , 'metas en relación con el lucimiento' (alfa $=0,65$ ).

Cuestionario de Estilos de Liderazgo (CELID) (Castro Solano, Nader \& Casullo, 2004): Corresponde a un cuestionario de 34 ítems que operacionalizan la teoría del liderazgo transformacional/transaccional propuesta por Bass y Avolio (1990). Este instrumento puede ser auto-administrado en forma individual o colectiva, respondiendo a frases relacionadas con el estilo de liderazgo transformacional (a través de las sub-dimensiones: carisma o influencia idealizada, inspiración, estimulación intelectual y consideración individualizada), el estilo transaccional (a través de las sub-dimensiones: recompensa contingente y dirección por excepción) y el estilo laissez faire. Los reactivos se responden mediante una escala de tipo Likert con 5 opciones de respuesta, cuyo escalamiento presenta las categorías Muy en desacuerdo/En parte en desacuerdo/Ni de acuerdo ni en desacuerdo/En parte de acuerdo/ Muy de acuerdo.

Para realizar el diseño y la validación del CELID, Castro Solano, Nader y Casullo (2004) llevaron a cabo un estudio de cuya muestra participaron militares y población general no consultante. Partiendo de la versión española del MLQ que disponía de 70 ítems, analizaron preliminarmente la correlación entre cada ítem y la escala con el propósito de eliminar los ítems con correlaciones ítem-escala bajas $(r<0,30)$; luego, llevaron a cabo un análisis factorial de componentes principales con rotación Varimax, cuya solución factorial presentaba siete factores 
que lograban explicar el 52,25\% de la varianza de las puntuaciones, quedando constituida la escala definitiva por 34 ítems. En un segundo momento, Castro Solano, Nader y Casullo (2004) realizaron un análisis factorial confirmatorio verificando la estructura para un modelo de tres factores (liderazgo transformacional, transaccional y laissez faire) en forma conjunta, y separada para los dos tipos de poblaciones estudiadas, resultando un ajuste aceptable para las dos poblaciones combinadas; el modelo de tres factores para población militar presentó un ajuste óptimo, con óptima validez discriminante entre los tres factores propuestos (Castro Solano, 2005). Por último, los mismos autores verificaron la fiabilidad del instrumento calculando el coeficiente alfa de Cronbach, obteniéndose fiabilidades bastante satisfactorias para cada una de las siete escalas, a saber: 'Población cadetes' $(n=111)$, liderazgo transformacional (carisma: 0,70; estimulación intelectual: 0,77; inspiración: 0,63; consideración individualizada: 0,70 ); liderazgo transaccional (recompensa contingente: 0,75 ; dirección por excepción: $0,76)$; laissez faire: 0,70 . 'Población oficiales' $(n=61)$, liderazgo transformacional (carisma: 0,66; estimulación intelectual: 0,71 ; inspiración: 0,60; consideración individualizada: 0,60 ); liderazgo transaccional (recompensa contingente: 0,62; dirección por excepción: 0,73); laissez faire: 0,75 . 'Población civil' $(n=191)$, liderazgo transformacional (carisma: 0,64; estimulación intelectual: 0,80; inspiración: 0,61 ; consideración individualizada: 0,60 ); liderazgo transaccional (recompensa contingente: 0,72; dirección por excepción: 0.70); laissez faire: 0,75. Estos índices de fiabilidad resultaron meritorios, tanto para población civil como para población militar, sin evidenciar significativas discrepancias en la evaluación comparada de ambas poblaciones (Castro Solano, 2005).

Cuestionario de Valores Contextualizados (VAL) (Castro Solano, 2005; Casullo \& Castro Solano, 2004): Este instrumento corresponde a una escala auto-administrable dispuesta de 30 ítems que evalúan los valores en dominios específicos para población adulta. Está basado en la tipología de diez valores de Schwartz et al. (2001) mencionada anteriormente, con el propósito de evaluar la orientación de los valores de las personas en la recreación y vida social, en las relaciones familiares y en el trabajo. Estas tres áreas de orientación y evaluación valórica constituyen formalmente las tres partes en que se presenta el instrumento, cada una de las cuales consta de diez ítems sobre los que la persona tiene que autoevaluarse en relación con el comportamiento descripto por cada frase (Castro Solano, 2005). ${ }^{2}$ Los ítems plantean situaciones que expresan determinados valores, las que el sujeto debe tratar de valorar según una escala de 1 a 5 de acuerdo con sus preferencias, expresando el número 5 su máxima preferencia y el 1 la menor; el número 3, señala un punto intermedio de preferencia en la escala.

Los diez tipos motivacionales de valores que define conceptualmente la teoría constituyen las dimensiones del instrumento, agrupándose en cuatro metas generales que deben tenerse en cuenta para interpretar los datos resultantes de la administración; a saber: apertura al cambio (que agrupa los valores de Estimulación, Autodirección y Hedonismo); conservación (valores de Tradición, Conformidad y Seguridad); trascendencia o autotrascendencia (valores de Universalismo y Benevolencia); por último, autopromoción (valores de Poder y Logro).

Para el diseño de la prueba VAL, Casullo y Castro Solano (2004) seleccionaron tres muestras intencionales correspondientes a población general de mujeres y varones adultos jóvenes de nivel educativo medio-alto, cadetes que cursaban sus estudios en una institución militar argentina y oficiales de carrera jóvenes que revistaban en las FF.AA. Inicialmente, los autores calcularon una puntuación promedio para cada uno de los diez valores en función de las respuestas a la escala en cada área, para población civil y militar (estudiantes y oficiales); luego, analizaron los valores que diferenciaban a los tres grupos muestrales a través de un análisis discriminante (resultando Tradición, Hedonismo y Autodirección los valores divergentes), agruparon las orientaciones de valores en las cuatro metas generales que propone Schwartz (2001) y estudiaron la existencia de divergencias en las prioridades de valores para cada contexto específico (recreación y vida social, relaciones familiares y trabajo) llevando a cabo análisis discriminantes cuyos resultados oscilaban entre el $45 \%$ y el $90 \%$ de la varianza. Por último, los

2. En el presente estudio, se administró solamente la Parte 3 del cuestionario, la relativa al Trabajo, dado que se decidió evaluar los valores de los estudiantes militares en el área laboral en que se desempeñarán en su futuro profesional. 
autores calcularon la consistencia de los valores a través del coeficiente alfa de Cronbach para cada una de las dimensiones que agrupan a los tipos de valores y para cada una de las poblaciones (civiles, cadetes y oficiales), permitiendo verificar que las dimensiones conservación y trascendencia resultaron las más consistentes para los tres tipos de poblaciones (rango entre 0,60 y 0,70 aproximadamente); contrariamente, las dimensiones autopromoción y apertura al cambio fueron las menos consistentes para los tres tipos de poblaciones (rango entre 0,50 y 0,60$)$, sin observarse grandes discrepancias (Castro Solano, 2005).

\subsection{Procedimiento}

Contando con el consentimiento de las autoridades del instituto (donde al momento de realizar la investigación los estudiantes cursaban los diversos niveles de formación académica de grado e instrucción militar), los sujetos participaron voluntariamente de la evaluación psicométrica, siendo administrada de forma grupal la batería de instrumentos descriptos en el anterior apartado. Para ello, se agrupó a la muestra de estudiantes en una sala de conferencias que contaba con asientos y apoyos de lápiz y papel individuales.

Antes de iniciar la consigna de trabajo, se aclaró a los participantes que si bien la prueba no sería de carácter anónimo (es decir que debían constar en el protocolo de administración su nombre y apellido), los datos recopilados serían utilizados a los fines de generalizar la información científicamente, y solamente los investigadores guardarían relación con los protocolos individuales, sin compartir los casos con las autoridades de la institución. Se informó a los sujetos que eventualmente podrían renunciar a su participación en cualquier momento del estudio. Tal procedimiento se llevó a cabo dado que (a los fines del análisis estadístico) se requería identificar individualmente a los participantes, para luego determinar su correspondencia con las calificaciones relativas a su rendimiento general promedio.

Los miembros del equipo de investigación estuvieron a cargo de la administración de la batería de test, cuya duración fue de aproximadamente una hora reloj. En la fase posterior, se llevaron a cabo las tareas de data entry (a cargo del equipo de investigación) y la recopilación de las calificaciones promedio para cada caso, para lo cual se requirió establecer contacto directo con el área de evaluación del instituto.

\subsection{Análisis de los datos}

Los datos fueron analizados mediante el soporte técnico estadístico que provee el paquete informático SPSS 11.0, llevando a cabo análisis de regresión lineal jerárquica. Para analizar los niveles críticos de significación asociados a tal coeficiente o medida estadística, se aceptó un error $\alpha<0,05$.

Preliminarmente, en relación a las variables principales sobre las cuales se focaliza el presente estudio, a saber, la inteligencia cultural, la personalidad, la motivación para el aprendizaje, el liderazgo y los valores humanos en el trabajo, se ha propuesto un criterio clasificatorio ad hoc a través del cual se integraron las cinco variables anteriormente mencionadas, consolidando un modelo de predictores globales del rendimiento de estudiantes militares que se bi-dimensionaliza mediante dos grupos de variables predictoras:

- Predictores actuales, representados por la inteligencia cultural, los factores de la personalidad y la motivación para el aprendizaje;

- Predictores prospectivos, integrados por la inteligencia cultural, los estilos de liderazgo y los valores humanos en el trabajo.

Vale aclarar, en principio, que dicha clasificación considera a la inteligencia cultural como una variable predictora del rendimiento de los estudiantes, tanto en el espacio actual (formativo-instructivo) como en el espacio futuro de accionar profesional, siendo esta variable, en el primer caso, forjada en la 'experiencia personal' (tanto en contextos civiles como militares) a través de vivencias o intercambios personales o grupales de índole multicultural, experimentados en áreas locales o en otras naciones por diferentes propósitos (viajes de estudio, motivos laborales personales o familiares, competencias deportivas, visitas, vacaciones, etc.), para posteriormente desplegarse quizás con mayor intensidad profesional, cuando el flamante subteniente $u$ oficial lleve a cabo operaciones militares de comando o conducción en contextos multiculturales diversos, ya sea en ocasión de ser destinado a cuarteles dependientes 
del Ejército ubicados en diferentes provincias argentinas, participe de viajes protocolares 0 de intercambios comunicacionales locales que requieran el establecimiento de relaciones con visitantes o agregados militares extranjeros que se acerquen a unidades militares argentinas, o bien, voluntariamente, lleve a cabo misiones de paz de carácter internacional. Por ambos motivos actuales y prospectivos, es que se ha considerado a la inteligencia cultural como un predictor del rendimiento del estudiante, cuya relevancia emerge en ambos momentos temporales del proceso de formación e instrucción profesional, por motivos conceptuales y pragmáticos.

Asimismo, este subgrupo de predictores actuales del rendimiento, que supone a la inteligencia cultural como una variable de relevante jerarquía en relación al primero (más que nada por su doble participación temporal), se halla integrado por los factores de la personalidad, los cuales constituyen el modo de ser singular de los estudiantes a través de los cuales se dinamizan las acciones que subyacen a su historia personal, el carácter individual y el temperamento, siendo éstos elementos constitutivos de dicho predictor; al tiempo que la motivación para el aprendizaje, corresponde a un elemento de relevancia en lo que respecta a los intereses personales por recibir la formación académica o bien su preferencia por la instrucción militar, que el cadete despliega sobre los procesos de aprendizaje y sobre los ejercicios de instrucción o entrenamiento (ya sea a nivel áulico o sobre el terreno, determinando su rendimiento sobre las áreas y núcleos específicos).

Por otro lado, el subgrupo correspondiente a los predictores prospectivos del rendimiento, se halla integrado ad hoc por la inteligencia cultural (por los motivos recientemente explicados) junto a los estilos de liderazgo, que radican en la experiencia individual de cada cadete en calidad de 'seguidor' de su líder (el oficial instructor, por ejemplo), cuyos efectos de éstos modelos de liderazgo implícitos forjarán cognitivamente su estilo de conducción, al tiempo que sus primeras experiencias como Jefe de Grupo o Subinstructor durante el tercer año y Jefe de Sección Básica e Instructor (supervisado) durante el cuarto año de la carrera, también contribuirán a la construcción del estilo de conducción militar del estudiante. Por último, los valores humanos en el trabajo, se consideran en este estudio posibles predictores futuros del rendimiento del cadete, puesto que se ha limitado el área de abordaje y aplicabilidad de dicha variable, considerando en la evaluación de la muestra aquellas situaciones valóricas relacionadas directamente con su 'potencial' trabajo profesional, obviando otras relativas a aspectos de recreación, vida social y relaciones familiares; los valores personales, atravesados por los valores corporativos que constituyen la cultura organizacional, y que también influirán sobre las futuras prácticas del liderazgo (Schein, 1988), orientan la formación e instrucción actual, contribuyendo a cristalizar un conjunto de valores profesionales a través de los cuales el futuro líder militar operará en el contexto de trabajo intra y/o intercultural. Por dichos motivos, es que los estilos de liderazgo y los valores humanos centrados hacia el ámbito de la práctica laboral-profesional, se legitiman como factores predictores del rendimiento del cadete en una dirección prospectiva de su desempeño; vale decir, elementos de suma importancia para la praxis profesional futura más que para su rendimiento actual, puesto que aún no son profesionales líderes ni trabajan como tales.

\section{RESULTADOS}

\subsection{Análisis de predictores actuales del rendimiento general}

A fin de dar respuesta al primer objetivo planteado en el estudio, tendiente a analizar jerárquicamente en qué grado la inteligencia cultural, la personalidad y la motivación para el aprendizaje resultan predictores del rendimiento general de los estudiantes, se comparó el grado de interacción predictiva que existe entre tales variables en relación al promedio general del año alcanzado por los cadetes. Para ello, se realizó un análisis estadístico de Regresión Lineal, jerarquizando los tres bloques de variables predictoras mediante tal procedimiento, a fin de respetar el mencionado ordenamiento por jerarquías establecido ad hoc.

En principio, estos resultados permiten observar que la inteligencia cultural reflexivamotivacional constituye un factor predictor del rendimiento general de los estudiantes. 
Inicialmente, en el análisis del primer modelo predictivo, integrado por un único bloque correspondiente a las tres dimensiones que constituyen la inteligencia cultural, la dimensión cognitiva había presentado el mayor Coeficiente Estandarizado Beta, aunque éste no resultase significativo $(\beta=0,095 ; p=0,099)$, en tanto la dimensión reflexiva-motivacional presentaba una menor tendencia significativa $(\beta=0,084 ; p=0,133)$, incluso comparada a la inteligencia cultural conductual-lingüística $(\beta=0,070 ; p=0,169)$. No obstante, se observó que el Modelo 2, en el que se incluía el bloque correspondiente a los factores de la personalidad, presentó un incremento en el nivel de significación asociado al Coeficiente Estandarizado Beta obtenido para la inteligencia cultural reflexiva-motivacional $(\beta=0,117 ; p=0,065)$, resultando dicho factor el de mayor tendencia jerárquica predictiva en lo que a la inteligencia cultural respecta; en tanto la dimensión conductual-lingüística $(\beta=0,085 ; p=0,096)$ y cognitiva $(\beta=0,092$; $p=0,103)$ no evidenciaron significación alguna, la inteligencia reflexiva-motivacional presentó cierta tendencia hacia el nivel de significación crítico asociado que el presente estudio ha considerado para los coeficientes estadísticos analizados $(p<0,05)$, (Tabla 1). Por último, para el Modelo 3 en el cual se adicionaba el bloque correspondiente a las dimensiones de la motivación para el aprendizaje, el análisis arrojó para la inteligencia cultural reflexiva-motivacional un Coeficiente Estandarizado Beta significativo

Tabla 1:

Predictores actuales del rendimiento general de los cadetes del Colegio Militar de la Nación, cursantes de la Licenciatura en Conducción y Gestión Operativa encuestados en agosto-setiembre del año 2008.

Resumen del modelo

\begin{tabular}{|c|c|c|c|c|c|c|c|c|c|}
\hline \multirow[b]{2}{*}{ Modelo } & \multirow[b]{2}{*}{$\mathbf{R}$} & \multirow[b]{2}{*}{ R cuadrado } & \multirow[b]{2}{*}{$\begin{array}{c}\text { R cuadrado } \\
\text { corregida }\end{array}$} & \multirow[b]{2}{*}{$\begin{array}{c}\text { Error típ. de la } \\
\text { estimación }\end{array}$} & \multicolumn{5}{|c|}{ Estadisticos de cambio } \\
\hline & & & & & $\begin{array}{l}\text { Cambio en } \mathrm{R} \\
\text { cuadrado }\end{array}$ & Cambio en $\mathrm{F}$ & gl1 & $\mathrm{g} \mid 2$ & $\begin{array}{c}\text { Sig. del cambio } \\
\text { en } \mathrm{F}\end{array}$ \\
\hline \multirow{3}{*}{$\begin{array}{l}1 \\
2 \\
3\end{array}$} & ,180(a) &, 032 &, 025 & 8,124784 &, 032 & 4,418 & 3 & 396 &, 005 \\
\hline &, $320(b)$ & , 102 &, 084 & 7,874772 & .070 & 6,109 & 5 & 391 &, 000 \\
\hline &, $365(c)$ & , 133 & 109 & 7,769074 & 031 & 4,570 & 3 & 388 &, 004 \\
\hline
\end{tabular}

a. Variables predictoras: (Constante), conductual-linguistica, refexiva-motivacional, cognitiva. b. Variables predictoras: (Constante), conductual-lingüistica, reflexiva-motivacional, cognitiva, neuroticismo, extraversión, afabilidad.

conciencia, apertura a la experiencia.
c. Variables predictoras: (Constante), conductual-lingüistica, reflexiva-motivacional, cognitiva, neuroticismo, extraversión, afabilidad, conciencia, apertura a la experiencia, metas de lucimiento, metas aversivas externas, metas de dominio.

Coeficientes(a)

\begin{tabular}{|c|c|c|c|c|c|c|c|c|}
\hline \multirow[b]{2}{*}{ Modelo } & & \multicolumn{2}{|c|}{$\begin{array}{l}\text { Coeficientes no } \\
\text { estandarizados }\end{array}$} & \multirow{2}{*}{$\begin{array}{c}\begin{array}{c}\text { Coeficientes } \\
\text { estandarizados }\end{array} \\
\text { Beta }\end{array}$} & \multirow[t]{2}{*}{$t$} & \multirow[t]{2}{*}{ Sig. } & \multicolumn{2}{|c|}{$\begin{array}{c}\text { Estadisticos de } \\
\text { colinealidad }\end{array}$} \\
\hline & & $\mathrm{B}$ & Error típ. & & & & Tolerancia & FIV \\
\hline \multirow[t]{4}{*}{1} & (Constante) & 67,812 & 2,689 & & 25,216 &, 000 & & \\
\hline & reflexiva-motivacional & 1,072 & 711 & .084 & 1,506 & 133 & .778 & 1,286 \\
\hline & cognitiva &, 940 &, 569 & 095 & 1,652 & 099 & 742 & 1,347 \\
\hline & conductual-lingũistica & 650 & 472 & 070 & 1,377 & 169 & 948 & 1,055 \\
\hline \multirow[t]{9}{*}{2} & (Constante) & 65,299 & 5,638 & & 11,582 &, 000 & & \\
\hline & reflexiva-motivacional & 1,484 & 803 & 117 & 1,848 & .065 & .573 & 1,744 \\
\hline & cognitiva & 911 & .557 & .092 & 1,635 & 103 & .727 & 1,376 \\
\hline & conductual-lingüistica & 788 & 473 & .085 & 1,667 & .096 & 889 & 1,125 \\
\hline & extraversión & $-9,489 E-02$ &, 738 &,- 008 &,- 129 & 898 & 635 & 1,576 \\
\hline & afabilidad & $-2,766$ & 843 & -193 & $-3,283$ & .001 & 667 & 1,500 \\
\hline & conciencia & 4,251 & 883 & 295 & 4,817 &, 000 & 614 & 1,628 \\
\hline & neuroticismo & 589 & 766 & 045 &, 768 & 443 & 677 & 1,477 \\
\hline & apertura a la experiencia & $-1,605$ & 945 &,- 104 & $-1,698$ & 090 & 609 & 1,641 \\
\hline \multirow[t]{12}{*}{3} & (Constante) & 74,495 & 6,171 & & 12,071 & .000 & & \\
\hline & reflexiva-motivacional & 1.652 & 814 & .130 & 2,030 & .043 & .543 & 1,841 \\
\hline & cognitiva & 959 & 556 & 097 & 1,725 & .085 & 711 & 1,407 \\
\hline & conductual-lingüistica & 874 & .470 & .094 & 1,861 & .063 & 876 & 1,141 \\
\hline & extraversión & -205 & 732 & -017 & -.280 & .779 & 629 & 1,589 \\
\hline & afabilidad & $-2,985$ & 838 & -208 & $-3,562$ & .000 & 656 & 1,525 \\
\hline & conciencia & 4,159 & 882 & 288 & 4,713 & .000 & 598 & 1,672 \\
\hline & neuroticismo & .746 & .758 & .057 & .983 & 326 & .673 & 1,486 \\
\hline & apertura a la experiencia & $-1,478$ & 944 & -096 & $-1,566$ & .118 & 595 & 1,681 \\
\hline & metas de dominio & $-1,137$ & .703 &,- 088 & $-1,619$ & 106 & .750 & 1,333 \\
\hline & metas de lucimiento &,- 705 & 467 & -.078 & -1.511 & 132 & 848 & 1,179 \\
\hline & metas aversivas externas & $-1,361$ & .520 &,- 139 & $-2,619$ & .009 & .792 & 1,262 \\
\hline
\end{tabular}

a. Variable dependiente: Promedio General del Año. 
$(\beta=0,13 ; p=0,043)$, lo cual evidencia que tal dimensión ha resultado predictora del rendimiento general de los estudiantes, a diferencia de la inteligencia cultural-lingüística que arrojó para este último modelo cierta tendencia de significación predictiva $(\beta=0,094 ; p=0,063)$ al tiempo que para la inteligencia cognitiva no resultó tal tendencia $(\beta=0,097 ; p=0,085)$.

En virtud de los resultados presentados en el párrafo anterior, la inteligencia cultural reflexiva-motivacional, es decir el monitoreo deliberado de afectos, conocimientos y habilidades (Thomas et al., 2008) que llevan a cabo los estudiantes a través de procesos psicológicos de los que participan sus representaciones mentales acerca de la diversidad cultural, resulta un predictor genuino de su rendimiento general.

Por otro lado, en lo que respecta a la variable personalidad, aquellos aspectos vinculados al factor responsabilidad o conciencia, es decir personas escrupulosas, ordenadas, con fuerte voluntad y determinación, puntuales y confiables (Castro Solano, 2005) resultan los predictores de mayor jerarquía significativa del rendimiento general $(\beta=0,288 ; p=0,000)$, al tiempo que la escasa afabilidad o agradabilidad manifiesta en el marco de las relaciones interpersonales a través de egocentrismo, insensibilidad, hostilidad, escepticismo respecto de las intenciones de los demás, dados en personas que resultan más competitivas que cooperativas, predicen el rendimiento general en forma negativa $(\beta=-0,208 ; p=0,000)$.

Por último, en cuanto a la motivación para el aprendizaje, las metas aversivas externas también han resultado predictores negativos del rendimiento general, lo cual significa que aquellos estudiantes que reciben la formación académica y estudian los respectivos contenidos motivados extrínsecamente por cuestiones tales como lograr el acceso a la carrera militar (la que implica necesariamente recibir la formación académica de forma paralela), como una obligación impuesta de la que tienen que liberarse en el menor tiempo posible y/o sólo para asegurar su permanencia en la institución, presentan una mayor probabilidad de alcanzar una baja performance $(\beta=-0,139 ; p=0,009)$.

\subsection{Análisis de predictores prospectivos del rendimiento general}

A fin de dar cumplimiento al segundo objetivo del estudio, en el cual se ha propuesto analizar los niveles de jerarquía en que la inteligencia cultural, los estilos de liderazgo y los valores humanos en el trabajo predicen el rendimiento general de los estudiantes, se comparó el grado de interacción predictiva que existe entre tales variables en relación al promedio general del año alcanzado por los cadetes, realizando nuevamente un análisis estadístico de Regresión Lineal a través del cual se han jerarquizado estos tres bloques de variables predictivas, en procura de respetar el ordenamiento por jerarquías establecido ad hoc.

Los resultados arrojados por tal procedimiento, han evidenciado que la inteligencia cultural cognitiva constituye un factor predictor del rendimiento general de los estudiantes, manifestando definitivamente tal significación en el tercer modelo predictivo $(\beta=0,126 ; p=0,034)$, a diferencia de las dos dimensiones restantes que no han presentado significación predictiva alguna en ninguno de los tres modelos.

Por otro lado, en cuanto a los estilos de liderazgo, el laissez faire ha operado como un predictor negativo del rendimiento general de los estudiantes $(\beta=-0,140 ; p=0,028)$, entendiéndose por tal resultado que la ausencia de liderazgo y la inactividad e ineficacia que esto conlleva, determina un bajo nivel de performance en los cadetes, más aún si se considera que éstos durante el tercer año de la carrera deberían hallarse en condiciones de asumir el rol de Jefes de Grupo y Subinstructores, y durante el cuarto año el rol de Jefes de Sección Básica e Instructores (bajo supervisión). No obstante, se observa una tendencia predictiva hacia el óptimo rendimiento general en lo que respecta a la sub-dimensión estimulación intelectual propia del estilo de liderazgo transformacional $(\beta=0,157 ; p=0,054)$, lo cual indicaría que aquellos cadetes que disponen de la capacidad para pensar nuevas soluciones o nuevas maneras de resolver problemas habituales (operando tal aspecto como un componte fundamental del liderazgo en situaciones críticas), seguramente vinculados a la formación académica, 
la instrucción militar, el entrenamiento físico y la potencial conducta profesional, alcanzan mayor eficacia en su performance (Tabla 2).

Por último, los valores humanos vinculados a la esfera laboral no han presentado niveles de significación predictivos de la performance de los estudiantes, resultando esto lógico y comprensible, dado que los sujetos se hallan en una etapa pre-profesional (o pre-laboral) en el marco de una institución educativa, pese a que en los sucesivos tramos de la carrera se les asignen roles que implican tareas de conducción.

\section{DISCUSIÓN}

Acorde a la relevancia que ha mostrado en el presente estudio el factor de la personalidad responsabilidad o conciencia, algunos autores destacan la importancia de la responsabilidad en lo que respecta a los procesos de aprendizaje. Rivière (1992) refiriéndose a la teoría social cognitiva y a la teoría de la auto-eficacia postulada por Bandura (1999; 2002), establece que tal aspecto individual participa activamente de los procesos de aprendizaje, a través de mecanismos auto-reguladores de carácter

Tabla 2.

Predictores prospectivos del rendimiento general de los cadetes del Colegio Militar de la Nación, cursantes de la Licenciatura en Conducción y Gestión Operativa encuestados en agosto-setiembre del año 2008.

Resumen del modelo

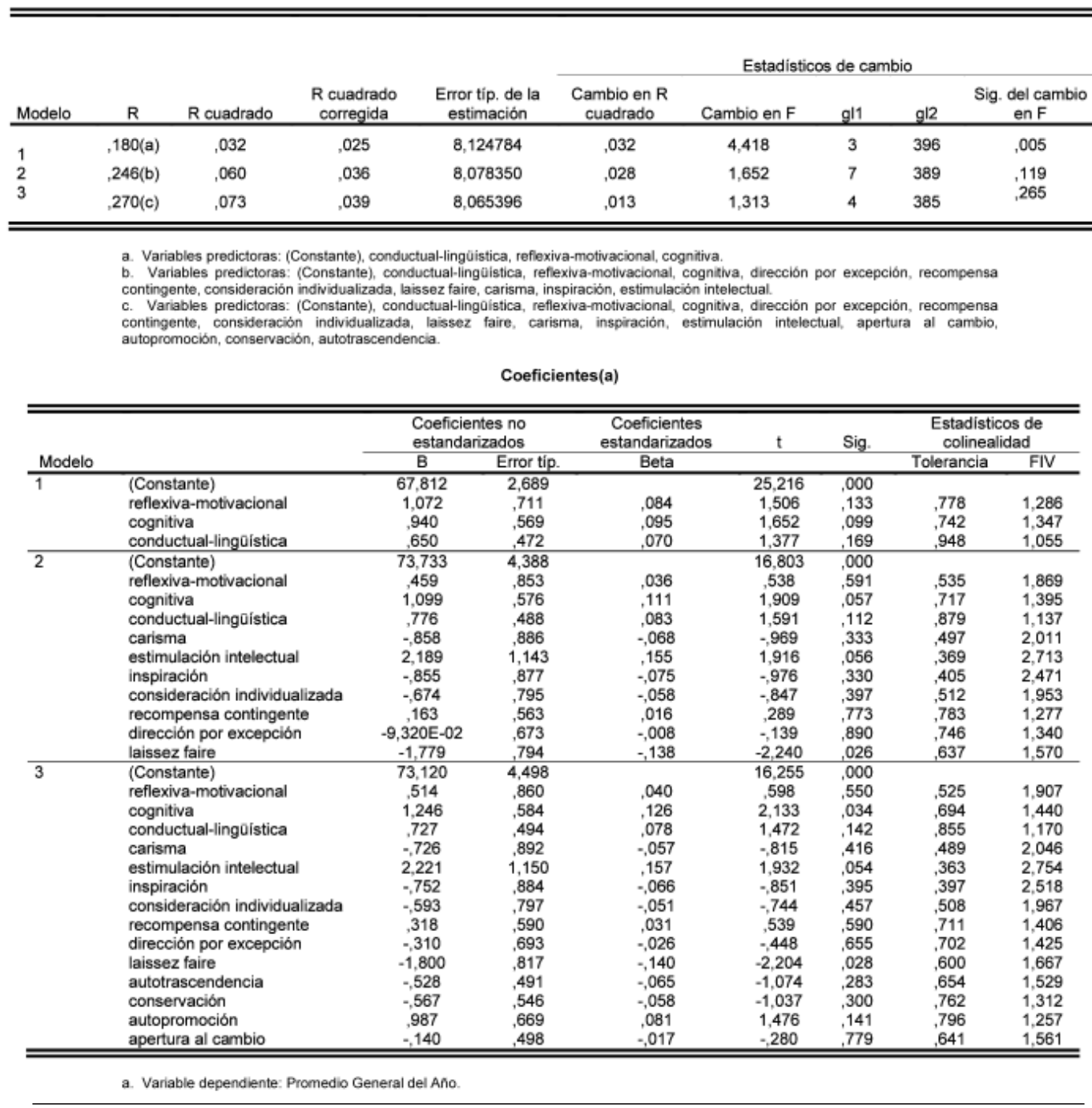


motivacional concediendo al pensamiento una importancia esencial en el control de la conducta. Bajo esta perspectiva de monitoreo auto-reflexivo sobre el propio acto de aprender, la metacognición cultural (Thomas et al., 2008) adquiriría su jerarquía predictiva en relación a la performance de los estudiantes, junto al factor conciencia propio de la personalidad. De forma tal que la responsabilidad de los cadetes -consolidándose como legítimos 'educandos' en relación a su propio proceso educativo- constituye a priori un elemento de gran importancia para la adquisición de conocimientos, más aún si los estudiantes practican cotidianamente la reflexión activa sobre tales procesos cognitivos; particularmente, la capacidad simbolizadora, la capacidad de previsión, la observación y el modelado a través de la capacidad vicaria (que otorga a docentes y oficiales instructores un lugar de gran relevancia educativa), la capacidad auto-reguladora (self-monitoring) y la capacidad de auto-reflexión, mecanizadas por la inteligencia cultural reflexiva-motivacional frente a la diversidad, deberían operar progresivamente durante la formación-instrucción de los futuros líderes militares. Nótese entonces la importancia que alcanza el pensamiento humano vinculado a la adquisición y despliegue de habilidades culturales con el auxilio de la inteligencia cultural, concebido éste como un instrumento adaptativo que incrementa la capacidad de enfrentarse de forma eficaz al entorno, dado que implica la representación y manipulación simbólica de los acontecimientos y sus interrelaciones (Rivière, 1992).

En lo que respecta a los estilos de liderazgo, el laissez faire predice significativamente el $14 \%$ del rendimiento general negativo de los cadetes, siendo el ciclo crítico en tal correlación significativamente negativo para el tercer año $(r=-0,206$; $p=0,039$ ) el cual corresponde al único año de cursada cuya correlación negativa resulta significativa para la performance, infiriéndose de ello que el cadete debe adaptarse al nuevo rol de Jefe de Grupo o Subinstructor, efectuando los primeros actos de conducción que lo acercan a su futuro profesional. Asimismo, la escasa afabilidad (expresada en conductas egocéntricas y poco cooperativas) que en este estudio predice negativamente la performance de los cadetes, podría generar un liderazgo ineficaz (Johnson y Hill, 2009).
Por tales motivos, se estima necesario dinamizar la reflexión y los conocimientos adquiridos como elementos que coadyuven a la construcción de un estilo de liderazgo transformacional para la resolución de situaciones críticas diversas, para efectivizar la toma decisiones en escenarios de gran incertidumbre cultural, etc. Para cumplir con tales premisas, es condición legítima fomentar la praxis cotidiana de una teoría constructiva del liderazgo que promueva la integración de la cultura operando ésta como un factor contextual sobre los modelos de liderazgo (Avolio, 2007; Depaula 2010).

\section{CONCLUSIONES}

El presente estudio ha permitido comprender y valorar la importancia que cobra el despliegue de inteligencia cultural en contextos formativos de liderazgo, en los cuales la personalidad y las metas de aprendizaje contribuyen significativamente en la dinámica de los procesos de adaptación cultural (Depaula, 2010).

Particularmente, alcanzando cierta consonancia con el modelo teórico presentado por Thomas et al. (2008), se destaca la importancia de la inteligencia cultural reflexiva-motivacional como factor de monitoreo de conductas culturales, la cual se activa con mayor intensidad frente a situaciones ambientales en los que se aproximan contextos de cambio cultural, de intensa dinámica afectiva y reajuste de los respectivos modelos mentales (como lo representan los primeros y últimos tramos de la carrera en cuestión; Depaula, 2010), donde se configuran escenarios de incorporación, transición y reinserción institucional, aculturación en definitiva (Berry, 1974; 1999).

Por otro lado, la inteligencia cultural cognitiva se consolida como un significativo predictor prospectivo positivo de la performance, facilitando la adquisición y aplicación de conocimientos específicos en dominios culturales, que cristalizados en las estructuras cognitivas del sujeto, serían necesarios para desplegar el accionar profesional futuro en línea con los requerimientos operacionales. Con todo, se expresa con mayor intensidad en etapas o ciclos de formación-instrucción en los cuales ya se ha alcanzado un óptimo nivel de adaptación 
al contexto diverso y se avecina un período de construcción cognoscitiva; empíricamente, esta cuestión encuentra explicación en el hecho de que dicha dimensión había alcanzado una correlación significativamente positiva sólo en la performance registrada para el tercer año de una Licenciatura en un instituto de formación militar.

En relación a los factores de la personalidad, vale destacar la jerarquizada influencia predictiva del factor conciencia sobre la performance de los estudiantes observada en el presente estudio, dado que coincide dimensionalmente con estudios precedentes similares (Goff \& Akerman, 1992; Rothstein, Paumonen, Rush \& King, 1994). En el marco de tales resultados, autores actuales enfatizan en que los institutos militares constituyen un sistema de educación especial difiriendo de otras instituciones de formación superior, dado que sus estudiantes deben tolerar un estricto entrenamiento militar adicional a los cursos académicos, pudiendo experimentar altos niveles de estrés físico y psicológico, desencadenantes de síntomas depresivos, por ejemplo (Lung, Lee \& Shu, 2006). Específicamente, Lung, Lee \& Shu (2002) sostienen que aquellos individuos con altos niveles de neuroticismo y baja extraversión presentan un alto riesgo de padecer desórdenes de ajuste a contextos militares. Al respecto, la muestra estudiada ha arrojado medias bajas tanto para el factor neuroticismo como para el factor extraversión, lo cual indicaría un escaso riesgo asociado a que los estudiantes del instituto en el cual se ha desarrollado la presente investigación presenten desórdenes de la personalidad en tal ámbito.

\section{REFERENCIAS}

Alonso Tapia, J. (1992). (Ed.), Motivar en la adolescencia. Teoría, evaluación e intervención. Madrid: Servicio de Publicaciones de la Universidad Autónoma.

Alonso Tapia, J. (1997). Orientación Educativa. Teoría, evaluación e intervención. Madrid: Síntesis.

Ang, S., Van Dyne, L., Koh, C., Ng, K. Y., Templer, K. J., Tay, Chandrasekar, N. A. (2007). Cultural Intelligence: Its measurements and effects on cultural judgments and decision making, cultural adaptation and task performance. Management and Organization Review, 3(3), 335-371.

Avolio, B. J. (2007). Promoting More Integrative Strategies for Leadership. Theory-Building. American Psychologist, 1(62), 25-33.
Bandura, A. (1999). (Ed.), Auto-Eficacia: Cómo afrontamos los cambios de la Sociedad actual. Bilbao: Desclée de Brouwer.

Bandura, A. (2002). Social cognitive theory in cultural context. Applied Psychology: An International Review, 51, 269-290.

Bartone, P. T. (1999). Personality Hardiness as a Predictor of Officer Cadet Leadership Performance. RTO MP$55,7,1-7$.

Bass, B. M. \& Avolio, B. (1990). Manual de Multifactor Leadership Questionnaire, Palo Alto, CA: Consulting Psychologists Press, Inc.

Berry, J. W. (1974). Psychological aspect of cultural pluralism: Unity and identity reconsidered. Topics in Culture Learning, 2, 17-22.

Berry, J. W. (1999). Intercultural Relations in Plural Societies. Canadian Psychology, 40(1), 12-21.

Braun, P., Wiegand, D. \& Aschenbrenner, H. (1991). The assessment of complex skills and of personality characteristics in military services. En R. Gal \& D. Mangelsdorff (Comps.), Handbook of Military Psychology (pp. 37-61). Nueva York: Wiley and Sons.

Castro Solano, A. \& Casullo, M. M. (2001). Rasgos de personalidad, rendimiento académico y bienestar autopercibido en adolescentes argentinos. Interdisciplinaria, 18(1), 65-85.

Castro Solano, A. \& Casullo, M. M. (2002). Razonamiento, motivación, intereses profesionales y rendimiento académico en cadetes militares. Revista Iberoamericana de Evaluación y Diagnóstico Psicológico, 13(1), 83-101.

Castro Solano, A. (2005). Técnicas de evaluación psicológica en los ámbitos militares. Buenos Aires: Paidós.

Castro Solano, A., Nader, M. \& Casullo, M. M. (2004). La evaluación de los estilos de liderazgo en población civil y militar argentina. Un estudio con el MLQ de Bass y Avolio. Revista de Psicología de la Universidad Católica del Perú, 22(1), 64-88.

Casullo, M. M. \& Castro Solano, A. (2004). Valores humanos y contextos en población civil y militar. Acción Psicológica (UNED), 3(1), 21-30.

Depaula, P. (2010). Integración de predictores globales del rendimiento en estudiantes de un instituto militar. Desarrollos y aplicaciones de inteligencia cultural para la defensa. (Tesis de Maestría). Universidad Abierta Interamericana. Bs As, Argentina.

Earley, P. C. \& Ang, S. (2003). Cultural intelligence: Individual interactions across cultures. CA: Stanford University Press.

Goff, M. \& Akerman, P. (1992). Personality-intelligence relations: Assessment of typical intellectual engagement. Journal of Educational Psychology, 84, 537-552.

Graen, G. B. \& Hui, C. (1999). U.S. Army leadership in the twenty-first century: challenges and implications for training. En J. G. Hunt, G. E. Dodge \& L. Wong (Eds.), Out-of-the-box leadership: Transforming the twenty-first century army and other top performing organizations (pp. 239-252). Stamford, Connecticut: Jai Press Inc.

Gurstein, M. (1999). Leadership in the peacekeeping Army o the future. En J. G. Hunt, G. E. Dodge \& L. Wong (Eds.), Out-of-the-box leadership: Transforming the twenty-first century army and other top performing organizations (pp. 195-218). Stamford, Connecticut: 
John, O. P. (1990). The Big Five' factor taxonomy: Dimensions of personality in the natural language and in questionnaires. En L. A. Pervin (Comp.), Handbook of personality: Theory and research. Nueva York: Guilford.

Johnson, J. L. \& Hill, W. R. (2009). Personality Traits and Military Leadership. Individual Differences Research, 1(7), 1-13.

Lung, F. W., Lee, F. Y. \& Shu, B. C. (2002). The relationship between life adjustment and parental bonding in military personnel with adjustment disorder in Taiwan. Military Medicine: An International Journal, 167, 678-682.

Lung, F. W., Lee, F. Y. \& Shu, B. C. (2006). The Premorbid Personality in Military Students With Adjustment Disorder. Military Psychology, 18(1), 77-88.

McFarland, M. (Marh-april, 2005). Military cultural education. Military Review, 62-69.

Ng, K. Y., Ramaya, R., Teo, T. M. S. \& Wong, S. F. (2005). Cultural Intelligence: Its potencial for military leadership development. Recuperado de: http://www.imta.info/PastConferences/Papers. aspx?ShowAll=True

Rivière, A. (1992). La teoría social del aprendizaje. Implicaciones educativas. En: C. Coll, J. Palacios \& A. Marchesi (Comps.), Desarrollo psicológico y Educación II, (pp.1-10). Madrid: Alianza.

Rothstein, M., Paumonen, S., Rush, J. \& King, G. (1994). Personality and cognitive ability predictors of performance in Graduate Bussiness School. Journal of Educational Psychology, 86(4), 516-539.

Rubinstein, R. A. (2003). In Practice: Cross-cultural considerations in complex peace operations. Negotiation Journal, 19, 29-49.
Schein, E. H. (1988). La cultura empresarial y el liderazgo. Una visión dinámica. Barcelona: Plaza \& Janes Editores.

Schwartz, S., Melech, G., Lehman, A., Burgess, S., Harris, M. \& Owens, V. (2001). Extending the cross-cultural validity of the theory of basic human values with a different method of measurement. Journal of Cross Cultural Psychology, 32(5), 519-542.

Schwerzel, J. (2005). Transforming attitudes. NATO Review: NATO and peace-building. Recuperado de: http:// www.nato.int/docu/review/2005/issue2/english/ art3_pr.html

Ng, K.Y., Ramaya, R., Teo, T.M.S. \& Wong, S.F. (2005) Cultural Intelligence: Its potencial for military leadership development. Recuperado de: http://www.imta.info/ PastConferences/Papers.aspx?ShowAll=True

Shamir, B. \& Ben-Ari, E. (1999). Leadership in an open army. En J. G. Hunt, G. E. Dodge \& L. Wong (Eds.), Outof-the-box leadership: Transforming the twenty-first century army and other top performing organizations (pp. 15-40). Stamford, Connecticut: Jai Press Inc.

Skelton, I. \& Cooper, J. (2005). You're are not from here, are you? Joint Force Quarterly Issue, 36, 12-16.

Thomas, D. C., Elron, E., Stahl, G., Ekenlund, B. Z., Ravlin, E. C., Cerdin, J., et al. (2008). Cultural Intelligence: Domain and Assessment. International Journal of Cross Cultural Management, 2(8), 123-143.

Yukl, G. (1999). Leadership competencies required for the new Army and approaches. En J. G. Hunt, G. E. Dodge \& L. Wong (Eds.), Out-of-the-box leadership: Transforming the twenty-first century army and other top performing organizations (pp. 255-276). Stamford, Connecticut: Jai Press Inc.

Para citar este artículo:

Depaula, D. (2012). Predictores globales de la performance de estudiantes militares. Ciencias

Psicológicas VI (2): 135-148. 